\title{
Use of monoclonal antibodies to detect disease associated HLA-DRB1 alleles and the shared epitope in rheumatoid arthritis
}

\author{
Ian Wicks, Geoff McColl, Angela D’Amico, Loretta Dougherty, Brian Tait
}

\begin{abstract}
Objective-To use a panel of monoclonal antibodies (Mab) which recognise HLA class II alleles associated with rheumatoid arthritis for fluorescence activated cell sorter (FACS) analysis of peripheral blood mononuclear cells (PBMNC) from patients with early and established rheumatoid arthritis and to compare these results against DNA oligotyping of HLA class II molecules in the same patients.

Methods-27 patients (18 from an early arthritis clinic, nine with established rheumatoid arthritis) were studied using both techniques. PBMNC were stained with Mab which recognise the shared epitope, the HLA-DRB ${ }^{\star}{ }^{\star 04}$ molecule and its $\star_{0401},{ }^{\star} 0404$ subtypes in the presence of bound peptide. Mab stained cells were analysed by FACS. Genomic DNA was prepared from PBMNC and used for DNA oligotyping and sequencing by standard methods.

Results-FACS analysis of Mab stained PBMNC gave identical results to those obtained by DNA oligotyping in 26/27 patients. The antibodies identified the shared epitope in 14/14 cases and the presence of an HLA-DRB $1{ }^{\star} 04$ molecule in $12 / 12$ cases. HLA-DRB ${ }^{\star}{ }^{0404}$ was identified in $4 / 4$ cases. HLA-DRB ${ }^{\star} 0401$ was identified in $5 / 6$ cases. One patient oligotyped as HLA-DRB1*0401, but consistently failed to react with the $\star_{0401}$ Mab. DNA sequencing of the second exon of the HLA-DRB $1{ }^{\star} 0401$ allele in this patient confirmed a normal HLADRB1*0401 genotype.

Conclusions-FACS analysis of PBMNC stained with Mab recognising the shared epitope and rheumatoid arthritis associated HLA susceptibility molecules provides a rapid, reliable, and more accessible alternative to DNA oligotyping. The apparent discordance between phenotypic and genetic analysis of HLA-DRB1*0401 in one patient, may reflect variability in HLADRB1*0401 gene expression or in class II peptide presentation.
\end{abstract}

(Ann Rheum Dis 1997;56:135-139)

Rheumatoid arthritis is a common but clinically heterogeneous disease. Recent work suggests that much of the joint damage in more severe cases occurs in the first few years of the disease. ${ }^{1}$ Accordingly, there is a trend towards earlier and more aggressive therapeutic intervention. Early stratification of patients who are at risk for severe disease is an important clinical issue for these reasons. Many studies have confirmed an association between rheumatoid arthritis and a restricted number of alleles at the highly polymorphic HLA-DRB1 locus (primarily $\mathrm{DRB} 1^{\star} 0401$, ${ }^{\star} 0404$, and $\left.{ }^{\star} 0101\right) .{ }^{23}$ Each of these alleles shares sequence homology in the third hypervariable region of the $\beta$ chain, which is referred to as the shared epitope. In a study of 201 patients with rheumatoid arthritis, who were unselected for disease severity, the presence of a single copy of ${ }^{\star} 0401,{ }^{\star} 0404$, or ${ }^{\star} 0101$ was associated with an odds ratio for rheumatoid arthritis of 3.5. ${ }^{4}$ Two identical copies of these alleles gave an odds ratio of 7.8 and this increased to 25.6 with ${ }^{\star} 0401 /{ }^{\star} 0404$ compound heterozygosity. Similar results were obtained from an analysis of 120 patients presenting with early arthritis. ${ }^{5}$ In this study, 10/11 compound heterozygotes $\left({ }^{\star} 0401 /{ }^{\star} 0404\right)$ developed erosions at one year. Conversely, patients without the HLA-DRB1 susceptibility alleles are at low (10-20\%) risk for progressive rheumatoid disease. ${ }^{23}$ These data raise the possibility of using HLA typing, together with other clinical and laboratory indices, to compile a profile of the patient with early inflammatory arthritis who is at risk for more severe rheumatoid disease.

HLA analysis has traditionally been performed in the tissue typing laboratory, initially using serological and cellular typing reagents and more recently with molecular techniques. The standard method for determining HLA-DR and its subtypes now involves isolation of genomic DNA from peripheral blood cells, the polymerase chain reaction (PCR) with sequence specific oligonucleotide primers and dot blotting, followed by hybridisation with probes directed at hypervariable region sequence differences. These molecular techniques, which are generally carried out in the context of HLA matching for organ transplantation, require specialised expertise and equipment and stringent quality control. While HLA typing has been used as a research tool in the study of rheumatoid arthritis, it has not been generally accessible as an aid to clinical management.

Recently, monoclonal antibodies (Mab) which recognise HLA-DR4, its subtypes ${ }^{\star} 0401$ and ${ }^{*} 0404$, and the shared epitope (QKAA/QRAA) have been generated. ${ }^{67}$ These antibodies are conformationally dependent
Accepted for publication 4 September 1996 
and require peptide loading of the $\mathrm{MHC}$ molecule. ${ }^{6}$ Fluorescence activated cell sorter (FACS) analysis of peripheral blood cells potentially offers a faster and more accessible method for identifying patients carrying rheumatoid arthritis associated HLA-DRB1 alleles early in the course of the disease. In this study, we compared the results of FACS analysis and genotyping in 27 patients, 18 of whom came from an early inflammatory arthritis clinic and nine had established rheumatoid arthritis.

\section{Methods}

SUBJECTS

Eighteen consecutive patients, all of whom fulfilled the 1987 revised American Rheumatism Association (ARA) criteria for rheumatoid arthritis, were enrolled from a study of early inflammatory polyarthritis conducted at the Royal Melbourne Hospital (RMH). Patients came from the community of suburban Melbourne. Nine patients with established rheumatoid arthritis-drawn from the RMH outpatient rheumatology clinic-were also studied.

ANTIBODY STAINING

Peripheral blood was collected into heparinised tubes and the mononuclear cells isolated on a LeucoSep Ficoll density gradient (Oropharma AG, Zurich). Peripheral blood mononuclear cells $\left(\right.$ PBMNC, $2 \times 10^{6}$ ) were stained with $30 \mu \mathrm{l}$ of irrelevant mouse immunoglobulin, Mab NFLD.D1 (anti-DR4; B1*04), Mab NFLD.D2 (anti-shared epitope QKRAA/QRRAA), Mab NFLD.D11 (antiDR4Dw4; B1*0401), Mab NFLD.D13 (anti-DR4Dw14; B1*0404), or NFLD.M6 (Mab to monomorphic determinant on HLA-DR; positive control) (supplied as supernatants by Terra Nova Biotech, Newfoundland, Canada). In cellular enzyme linked immunosorbent assays (ELISA), the NFLD.D2 also showed limited binding to QARAA containing alleles. ${ }^{7}$ Fluorescein isothiocyanate (FITC) conjugated Mab anti-CD3 (1:40) (Dako, Denmark) was added to all tubes in order to demarcate clearly the HLA class II expressing B cells. Cells were held for 20 minutes on ice and washed once through fetal calf serum. Phycoerythrin conjugated goat antimouse Mab was added (Terra Nova Biotech, Newfoundland, Canada) and cells held on ice for 30 minutes. Cells were washed once and resuspended in $2 \%$ paraformaldehyde in phosphate buffered saline before flow cytometry.

FLOW CYTOMETRY

A Becton Dickinson FACScan and FACScan Research software (Becton Dickinson, CA, USA) were used for analysis of Mab stained cells. Lymphocytes were separated from monocytes and contaminating granulocytes using side-scatter and forward-scatter parameters. Fluorescence in the FITC channel was plotted against phycoerythrin for the gated lymphocyte population. $T$ cells were clearly separated from the HLA class II positive B cells by double staining (figure). Increased B cell fluorescence in the phycoerythrin channel was usually obvious from inspection, but quadrants were set on all samples, using goat anti-mouse (GAM) conjugated phycoerythrin alone (negative control) and NFLD.M6 (positive control) to estimate staining in the phycoerythrin channel. The following formula was used to quantify fluorescence in the phycoerythrin channel:

$\frac{(\% \text { Test })-(\% \text { Negative control })}{(\% \text { Pos control })-(\% \text { Neg control })} \times 100$

A figure of greater than $20 \%$ was recorded as positive staining. FACS results were analysed without knowledge of the genotype.

HLA DNA OLIGOTYPING

DNA was isolated using the method of Miller et $a l^{8}$ from the buffy coat of anticoagulated blood samples. Genomic DNA was amplified by the PCR, dot blotted, and probed with digoxygenin labelled (Boehringer Mannheim, Germany) oligonucleotides specific for HLADRB1 variable sequences. A two step procedure was used, consisting of PCR amplification of the DRB1 second exon using generic primers and hybridisation with probes providing a serologically equivalent DR assignment (including DR4 and DR0101/0102). Secondly, primers amplifying only the DR4 group of alleles were used in the PCR with genomic DNA and the product probed with a series of oligonucleotides which defined ${ }^{\star} 0401-{ }^{\star} 0422$. A similar procedure was used to define the DR1 alleles. Following hybridisation and stringent washing, bound probes were detected using an anti-digoxygenin-alkaline phosphatase conjugate and chemiluminescent substrate (Boehringer Mannheim, Germany). Hybridisation signals were visualised by exposure to $x$ ray film (Kodak XAR-5).

\section{HLA-DR4 1 SEQUENCING}

Primers flanking the second exon of the HLADR4 1 gene were used to PCR amplify a 300 base pair fragment from the genomic DNA of patient 15 . The PCR product was purified with a High Pure PCR product purification kit (Boehringer Mannheim, Germany) and sequenced in both directions using a Taq Dye deoxy terminator cycle sequencing kit (Perkin Elmer, CA, USA) and an ABI automated DNA sequencer (Perkin Elmer, CA, USA).

\section{Results}

The peripheral blood mononuclear cells from 18 patients with early inflammatory arthritis and nine patients with established rheumatoid arthritis were stained with antibodies recognising HLA-DRB $1{ }^{\star} 04$, its variants ${ }^{\star} 0401$ and ${ }^{\star} 0404$, and the shared epitope itself. Genomic DNA was extracted from the same sample and typed using PCR and sequence specific oligohybridisation with DNA probes. The figure shows representative FACS profiles and corresponding HLA genotypes. Increased 


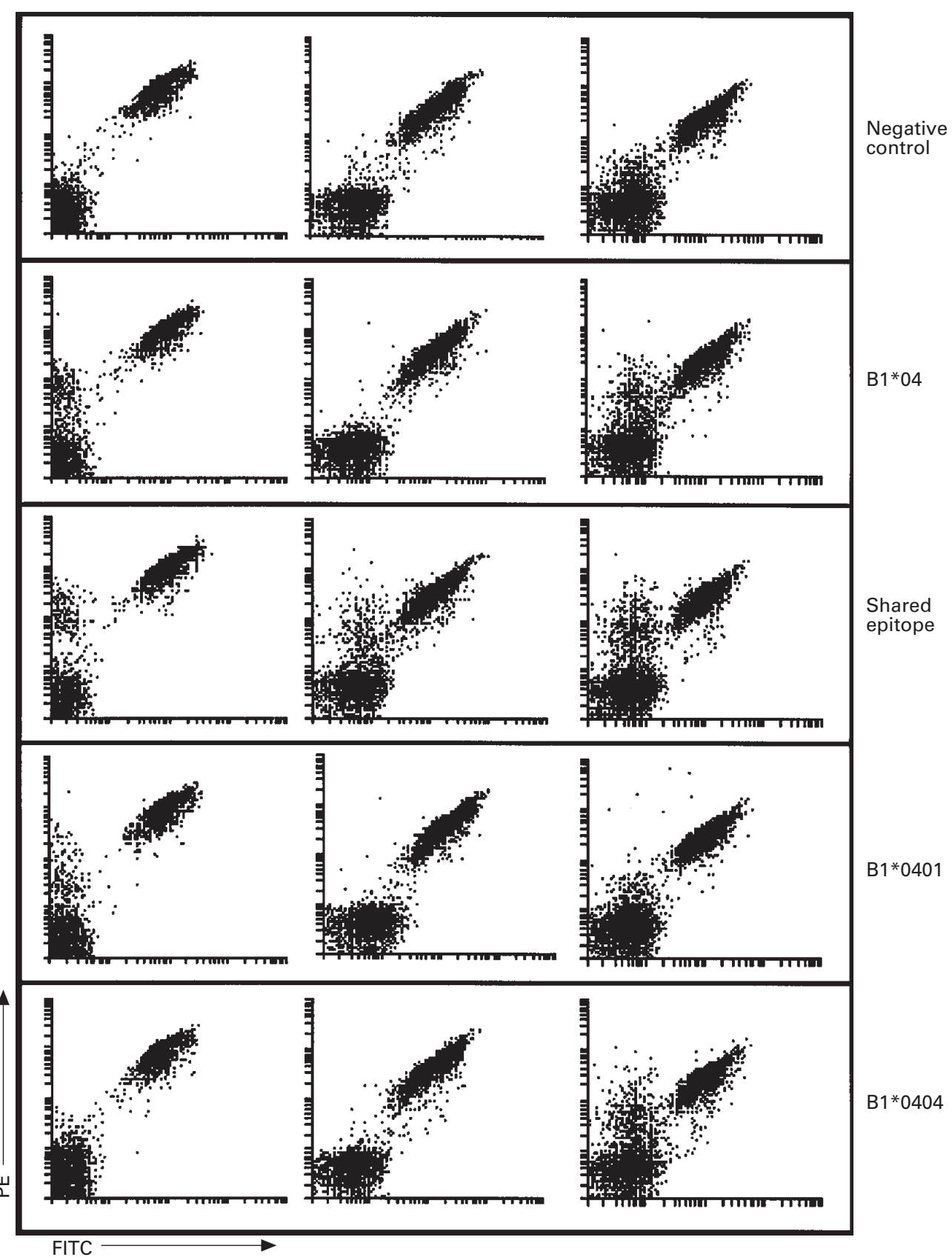

Two-colour FACS profiles of gated PBMNC stained with FITC conjugated murine anti-CD3 ( $X$ axis) and murine anti-HLA-DRB1 ${ }^{\star} 04$, shared epitope, ${ }^{\star} 0401,{ }^{\star} 0404$, or negative control antibodies (Y axis). Cells were then washed and stained with phycoerythrin conjugated goat anti-mouse antibody. Genotypes ( $\left.{ }^{\star} 0401,{ }^{\star} 0101,{ }^{\star} 0404\right)$ are listed above the figure. Anti-CD3 coated T cells also stain with the goat anti-mouse secondary antibody and are then clearly demarcated as a "double positive" population in the upper right quadrant. Positive HLA class II staining on B cells (in the lower left quadrant) is noted as a shift in fluorescence in the phycoerythrin channel.

fluorescence in the phycoerythrin channel (indicating positive staining) was usually obvious on inspection, but quadrants were carefully set on all samples, using the secondary antibody GAM-PE alone (negative control) and the Mab NFLD.M6, which recognises a non-polymorphic determinant on HLA-DR as a positive control, to estimate staining in the phycoerythrin channel. As shown in the table, the panel of Mab correctly recognised the relevant cell surface products encoded by the corresponding HLA genotypes in all but one of the patients with early inflammatory arthritis (patients 1-18). Patient 15 oligotyped as HLA$\mathrm{DRB} 1{ }^{\star} 0401 /{ }^{\star} 0101$, but failed to react with the ${ }^{\star} 0401$ antibody. Both the oligotyping and the FACS analysis were repeated on this patient and gave the same results. Sequencing of the second exon of the HLA-DRB ${ }^{\star}{ }^{\star} 04$ allele using genomic DNA as the substrate confirmed a normal ${ }^{\star} 0401$ genotype in this patient. The results of antibody staining were equivalent to HLA-DR oligotyping in nine patients (patients 19-27) with established rheumatoid arthritis. 
By FACS analysis, there was no cross reactivity of the NFLD.D2 Mab with alleles which might bear the QARAA epitope.

\section{Discussion}

Several recent studies have clearly established that HLA-DRB1 status contributes to the severity and outcome of rheumatoid arthritis $^{49^{10-11}}$ and is a useful prognostic marker in early disease. ${ }^{5}$ However, for a variety of reasons, HLA typing in rheumatoid arthritis has generally only been available in the research setting. Most tissue typing laboratories are necessarily preoccupied with typing for organ transplantation and it is doubtful that even limited HLA analysis would be routinely available for patients with early inflammatory arthritis. Ease of access and turnaround time from the laboratory are also important issues in clinical practice.

The present study was undertaken to evaluate the performance of a panel of Mab which recognise the HLA-DRB1 subtypes associated with rheumatoid arthritis. There was concordance between the results of FACS analysis of Mab stained PBMNC s and DNA oligotyping in $26 / 27$ cases. The antibodies identified the shared epitope in $14 / 14$ cases and the presence of the HLA-DRB ${ }^{\star} 04$ molecule in $12 / 12$ cases. All four HLA-DRB ${ }^{\star} 0404$ positive patients were correctly identified. Five out of six ${ }^{\star} 0401$ positive patients were correctly identified. One patient genotyped as HLADRB $1{ }^{\star} 0401$, but failed to react with the ${ }^{\star} 0401$ Mab (NFLD.D11). A similar discrepancy between HLA-DRB ${ }^{\star} 0401$ genotype and NFLD.D11 Mab reactivity has been encountered in one other patient, but would appear to be unusual (Marshall WH, personal communication). There are several possible explanations for this observation. All patients with established rheumatoid arthritis were correctly typed in spite of the long term use of disease

Results of FACS analysis with the HLA-DR Mabs compared with the genotype as determined by DNA oligotyping in 18 patients (1-18) with early inflammatory arthritis and in nine (19-27) with established rheumatoid arthritis

\begin{tabular}{|c|c|c|c|c|c|}
\hline Patient & $B 1^{\star} 04$ & $\begin{array}{l}\text { Shared } \\
\text { epitope }\end{array}$ & $B 1^{\star} 0401$ & $B 1^{\star} 0404$ & Genotype \\
\hline 1 & + & + & + & - & 0401,7 \\
\hline 2 & - & - & - & - & 2,10 \\
\hline 3 & + & + & - & - & 3,0405 \\
\hline 4 & + & + & - & - & 0403,0102 \\
\hline 5 & - & - & - & - & 7,13 \\
\hline 6 & - & - & - & - & 2,3 \\
\hline 7 & + & + & - & + & 0404,2 \\
\hline 8 & - & - & - & - & 3,11 \\
\hline 9 & - & + & - & - & 0101,2 \\
\hline 10 & - & - & - & - & 2,3 \\
\hline 11 & - & - & - & - & 11,2 \\
\hline 12 & + & + & + & - & 0401,0401 \\
\hline 13 & - & - & - & - & 07,0103 \\
\hline 14 & - & - & - & - & 2,3 \\
\hline 15 & + & + & - & - & 0401,0101 \\
\hline 16 & - & - & - & - & 2,3 \\
\hline 17 & - & - & - & - & 3,13 \\
\hline 18 & + & + & + & - & 0401,0401 \\
\hline 19 & + & + & + & + & 0401,0404 \\
\hline 20 & + & + & + & - & 0401,0101 \\
\hline 21 & - & + & - & - & 0101,0103 \\
\hline 22 & + & + & - & + & 0404,8 \\
\hline 23 & + & + & - & + & 0404,3 \\
\hline 24 & + & + & - & - & 0408,0101 \\
\hline 25 & - & - & - & - & 3,11 \\
\hline 26 & - & - & - & - & 2,7 \\
\hline 27 & - & - & - & - & 2,8 \\
\hline
\end{tabular}

modifying antirheumatic drugs (DMARD) and potential DMARD induced downregulation of HLA class II expression. NFLD.D11 reactivity has been shown to be critically influenced by the presence of bound peptide in the peptide binding site of the class II HLA molecule ${ }^{6}$ although the peptides involved are unknown. Antibody binding in this system could be influenced by the nature of the peptides available for presentation. Our HLA-DRB $1^{\star} 0401 /{ }^{\star} 0101$ patient whose PBMNC failed to stain with NFLD.D11 may have a disorder of peptide processing or loading in the HLA class II pathway. However, the positive staining for $\star_{0} 101$ would argue against a generalised peptide processing abnormality in this patient and might suggest reduced expression of the ${ }^{\star} 0401$ molecule itself. Variable transcription of HLA genes due to promoter polymorphisms has been described previously ${ }^{12}$ and is being further examined in our patient.

FACS analysis of Mab stained PBMNC is rapid and the results were usually available within a day of blood collection. Use of the antibodies and interpretation of results requires a FACS machine and a skilled operator, although both of these are now commonly available in routine pathology laboratories. A twofold increase in antibody staining due to DNA homozygosity (as occurred with patients 12 and 18 in our study) is not readily observable from the FACS profile and this is a relative disadvantage of the Mab method. A "double dose" of the rheumatoid arthritis associated susceptibility alleles is associated with more severe disease ${ }^{10}$ and is detected by DNA oligotyping. One possible use of the Mab method would therefore be to screen shared epitope positive patients in order to select those needing DNA oligotyping. However, compound heterozygosity (HLA-DRB $1^{\star} 0401 /$ ${ }^{\star} 0404$ ) seems to carry the worst prognosis in rheumatoid arthritis ${ }^{411}$ and this phenotype is readily identifiable using NFLD.D11 and NFLD.D13. We correctly identified the one HLA-DRB $1{ }^{\star} 0401 /{ }^{\star} 0404$ compound heterozygote (patient 19) in our study using the Mab panel.

Epidemiological studies using DNA oligotyping have established that several HLA class II DRB1 alleles, and in particular the shared epitope coded by these alleles, exert an important influence on the severity of rheumatoid arthritis. DNA oligotyping remains the gold standard for determining HLA status, but is not widely accessible and therefore has had limited impact on clinical management in diseases like rheumatoid arthritis. We believe simplified methods for detecting the shared epitope itself and rheumatoid arthritis associated HLA subtypes - such as the one outlined in this paper-will aid in the prompt identification of patients with early inflammatory arthritis who are at greater risk for severe rheumatoid disease. We thank Terra Nova Biotechnology for kindly supplying the
antibodies used in this study and the rheumatologists who antowed us to study their patients. We thank Michael Varney and allowed us to study their patients. We thank Michael Varney and
Carmel Kanaan from the Tissue Typing Laboratory of the 
Royal Melbourne Hospital for excellent technical assistance and Peter McNair for help with sample collection. We gratefully Reid Charitable Trusts and the Australian National Health and Medical Research Council.

1 Fuchs HA, Pincus T. Radiographic damage in rheumatoid arhtritis: description by nonlinear models. J Rheumatol arhtritis: description

2 Nepom GT, Nepom BS. Prediction of susceptibility to rheumatoid arthritis by human leukocyte antigen typing. Rheum Dis Clin North Am 1992;18:785-92.

3 Ollier W, Thomson W. Population genetics of rheumatoid arthritis. Rheum Dis Clin North Am 1992;18:741-58.

4 MacGregor A, Ollier W, Thomson W, Jawaheer D, Silman arthritis: increased association in men, young age at onset and disease severity. J Rheumatol 1995;22:1032-6.

5 Gough A, Faint J, Salmon M, Hassell A, Wordsworth P, Pilling D, et al. Genetic typing of patients with inflammatory arthritis at presentation can be used to predict outcome. Arthritis Rheum 1994;37:1166-70.

6 Drover S, Marshall WH, Kwok WW, Nepom GT, Karr RW. Amino acids in the peptide-binding groove influence an Amino acids in the peptide-binding groove influence an antibody-defined, disease associated
7 Drover S, Karr RW, Fu XT, Marshall WH. Analysis of monoclonal antibodies specific for unique and shared determinants on 1994;40:51-60.

8 Miller SA, Dykes DD, Ploesky HF. A simple salting out procedure for extracting DNA from human nucleated cells. Nucleic Acids Res 1988;16:1215.

9 Weyand CM, McCarthy TG, Goronzy JJ. Correlation between disease phenotype and genetic heterogeneity in rheumatoid arthritis. J Clin Invest 1995;95:2120-6.

10 Weyand CM, Hicok KC, Conn DL, Goronzy JJ. The influence of HLA-DRB1 genes on disease severity in rheumaence of HLA-DRB1 genes on disease severity in r
toid arthritis. Ann Internal Med 1992;117:801-6.

11 Wordsworth P, Pile KD, Buckley JD, Lanchbury JSS, Ollier $B$, Lanthrop M, et al. HLA heterozygosity contributes to susceptibility to rheumatoid arthritis. Am J Hum Genet 1992;51:585-91.

12 Balas A, Garcia-Sanchez F, Gomez-Reino F, Vicario JL. HLA class 1 allele (HLA-A2) expression defect associated with a mutation in its enhancer B inverted cat box in two families. Hum Immunol 1994;41:69-73.

13 Beaty JS, West KA, Nepom GT. Functional effects of a natural polymorphism in the transcriptional regulatory natural polymorphism in the transcriptional regulatory
sequence of HLA-DQB1. Mol Cell Biol 1995;15:477182 . 